\title{
Atomic resolution grain boundary analysis using atom probe tomography
}

\author{
P.J. Felfer, S.P. Ringer and J.M. Cairney \\ Australian Key Center for Microscopy and Microanalysis, The University of Sydney, NSW 2006, \\ Australia
}

Many of the properties of metallic materials are determined by the interfaces present within the microstructure, most importantly the grain boundaries and hetero-phase boundaries. In the last decades, the physical and chemical structure of interfaces in metals has been investigated in detail using electron microscopy. These investigations have yielded a great deal of knowledge about the physical structure, lately at an atomic level [1]. Yet, compared to structural information, relatively little is known about the local chemistry at grain boundaries due to the challenges conducting these types of experiments using transmission electron microscopy (TEM). Tomographic atom probes are able to identify single atoms by their time of flight, thus showing equal sensitivity throughout the periodic table. It has recently also been shown that atom probe experiments can achieve very high levels of spatial accuracy, quoted at $20 \mathrm{pm}$ in depth [2]. This advanced analysis technique enables the exploration of solute structures at and near interfaces at an atomic level even for elements that are only present in trace amounts.

Since the sample itself acts as the lens in the atom probe, crystalline imperfections (and the crystal structure itself) present sources of trajectory aberrations [3], which are thought to be the limiting factor for the resolution in atom probe tomography [2]. While in many cases this cannot be influenced, in the case of grain boundaries, aberrations have been shown to be minimal when the grain boundary is perpendicular to the measurement axis [4]. Even small tilts away from that condition lead to significant blurring of the interface.

In order to analyse grain boundaries at atomic resolution, samples must therefore be fabricated with the interface of interest oriented perpendicular to the tip axis. The utilization of a focused ion beam system with a micro-manipulator for lift-out can achieve this task [5]. In the present work, bars containing particular grain boundaries in a novel strip-cast steel (fig. 1a) have been extracted using a rapid and reproducible method. Specimens have been prepared from specifically selected boundaries including prior austenite boundaries (which are $\sim 500 \mu \mathrm{m}$ apart in this material) and other ferriteferrite boundaries. These bars have been distributed to create a number of samples ( 8-10), where each piece is welded onto a Mo-support structure and sculpted into a tapered needle shape. After locating the grain boundary in the TEM, the needles have been shortened so that the grain boundary is within $100 \mathrm{~nm}$ of the tip apex. $\mathrm{Ga}^{+}$damage has been minimised by using a $5 \mathrm{kV} \mathrm{Ga}$ beam, so that $15-20 \mathrm{~nm}$ of the sample length is removed. The resulting shape is depicted in fig. $1 \mathrm{~b}$.

Fig. 2 shows a TEM image and an atom probe image from the same prior austenite grain boundary in a strip-cast microalloyed steel. The results to be presented will be carried out at the lowest accessible temperature $(20 \mathrm{~K})$ and a pulse fraction of $20 \%$. This yields data of excellent quality, showing details of the crystal structure in the field desorption pattern. These patterns will be used to calibrate the reconstruction [6] and to obtain the crystallographic relationship between the two grains [7]. We will compare the solute levels approaching the grain boundary for the different types of grain boundaries. The reconstruction will show both the resolved lattice planes adjacent to the grain boundary and the hierarchy of the different solute atoms relative to the boundary. [8] 
References

[1] U. Dahmen et al., Phil. Trans. of the R. Soc. A 367 (2009) 3795

[2] B. Gault et al., Applied Physics Letters 95 (2009) 1

[3] M. Miller, Journal de Physique C6-11 (1987) 565

[4] D. Blavette et al., Acta Materialia 44-12 (1996) 4995

[5] K. Thompson et al., Ultramicroscopy 107-2/3 (2007) 2

[6] B. Gault et al., Journal of Applied Physics 105, 034913 (2009)

[7] P. Liddicoat et al., Materials Science Forum 618 (2009) 543

[8] This work was funded by the Australian Research Council (ARC). The authors are grateful for scientific and technical input and support from the Australian Microscopy \& Microanalysis Research Facility (AMMRF) node at the University of Sydney and financial support from the AMMRF travel and access program.
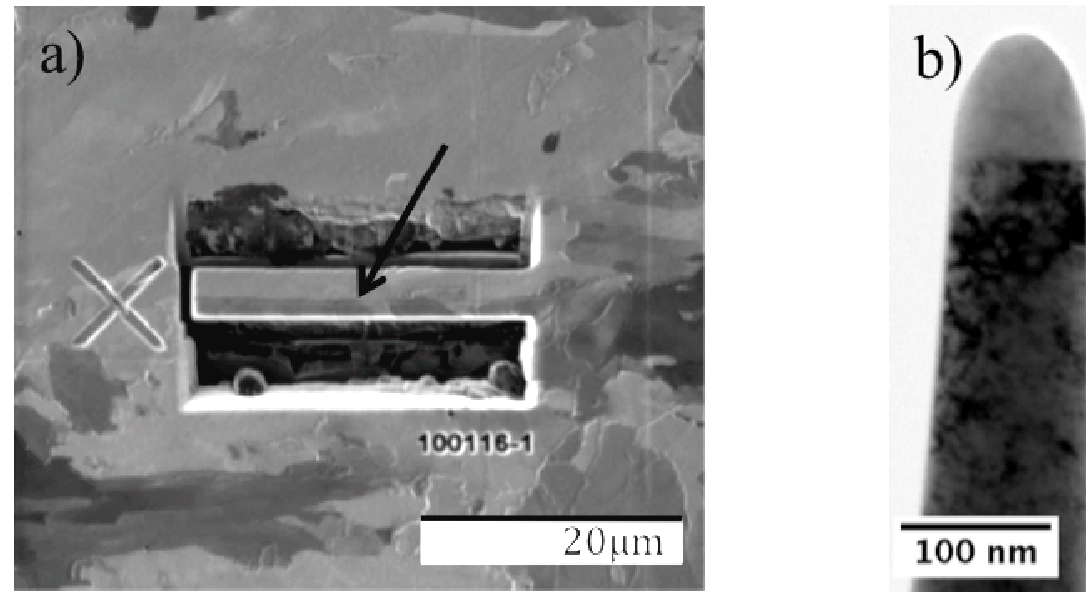

FIG. 1. Micrograph of a prior austenite grain boundary (arrowed), (a) prior to lift-out and (b) a TEM micrograph of the same specimen prepared by FIB and containing the boundary, ready for atom probe analysis.
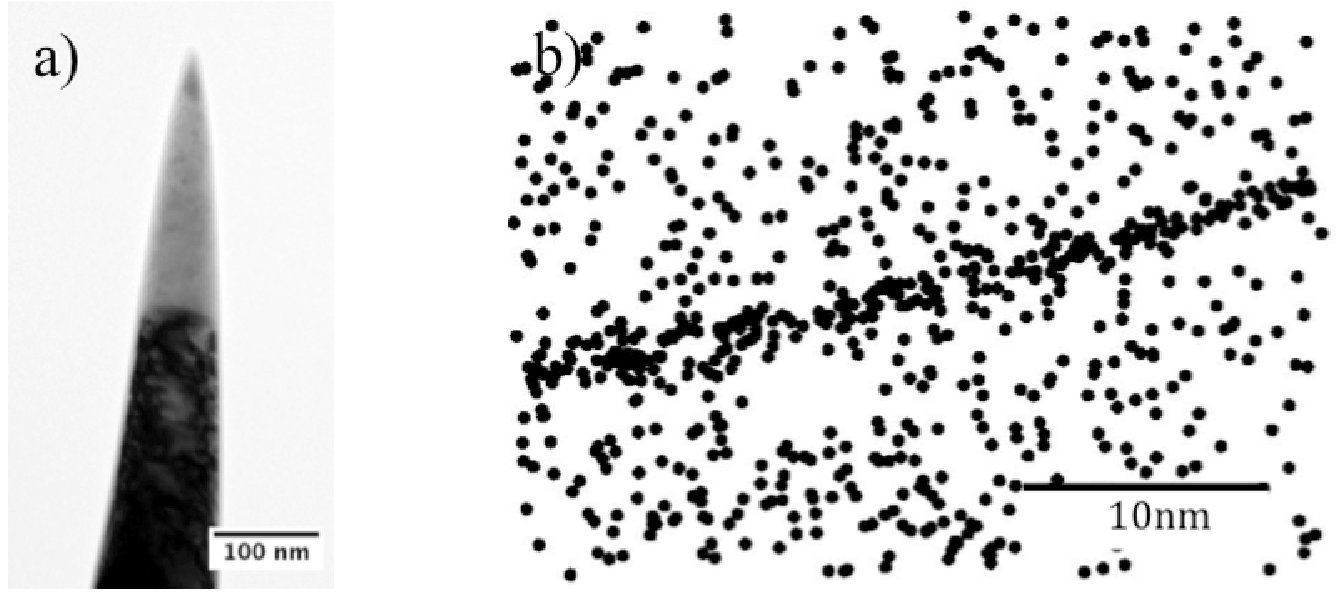

FIG. 2 (a) TEM micrograph of an atom probe specimen containing a prior austenite grain boundary and (b) the corresponding atom probe reconstruction showing the positions of the phosphorus atoms (30x30x20nm volume). The sample was shortened and shaped before the atom probe experiment. 